\title{
Mobility enhancement of strained Si transistors by transfer printing on plastic substrates
}

\author{
Wonho Lee ${ }^{1}$, Yun Hwangbo ${ }^{2}$, Jae-Hyun Kim ${ }^{2}$ and Jong-Hyun $\mathrm{Ahn}^{1}$ \\ Strain engineering has been utilized to overcome the limitation of geometric scaling in Si-based thin-film transistor (TFT) \\ technology by significantly improving carrier mobility. However, current strain engineering methods have several drawbacks: they \\ generate atomic defects in the interface between $\mathrm{Si}$ and strain inducers, they involve high-cost epitaxial depositions and they are \\ difficult to apply to flexible electronics with plastic substrates. Here, we report the formation of a strained Si membrane with \\ oxidation-induced residual strain by releasing a host $\mathrm{Si}$ substrate of a silicon-on-insulator (SOI) wafer. The construction of the \\ suspended $\mathrm{Si} / \mathrm{SiO}_{2}$ structures induces $>0.5 \%$ tensile strain on the top $\mathrm{Si}$ membrane. The fabricated TFTs with strained $\mathrm{Si}$ \\ channels are transferred onto plastics using a roll-based transfer technique, and they exhibit a mobility enhancement factor of \\ 1.2-1.4 compared with an unstrained Si TFT.
} NPG Asia Materials (2016) 8, e256; doi:10.1038/am.2016.31; published online 25 March 2016

\section{INTRODUCTION}

A critical path in the development of advanced silicon electronics involves a reduction in the dimensions of device geometries such as the channel thickness, channel length and gate dielectric thickness to increase the operating speed with lower power consumption and the density of integration in circuits. ${ }^{1-4}$ Continued performance improvement through geometric scaling has faced increasingly difficult challenges. ${ }^{5,6}$ Recent research focused on this issue has explored strain engineering that can increase the charge carrier mobility by creating tensile strain in Si with a capping stressor film, ${ }^{7}$ local stressors under channel $^{8}$ or embedded source/drain stressors. ${ }^{9,10}$ In particular, the introduction of an $\mathrm{Si}_{1-x} \mathrm{Ge}_{x}$ buffer layer with a large lattice constant represented by an epitaxial template can successfully generate biaxial tensile strain in $\mathrm{Si}^{11,12} \mathrm{The}^{11} \mathrm{Si}_{1-x} \mathrm{Ge}_{x}$ virtual buffer layer allows the fabrication of wafer-scale strained Si membranes ${ }^{13,14}$ and approximate doubling of the mobility enhancement factors of electrons in Si layers for fast, flexible electronic device applications. ${ }^{15-17}$ However, the process for introducing the $\mathrm{Si}_{1-x} \mathrm{Ge}_{x}$ buffer leads to undesirable misfit dislocations and interdiffusion of $\mathrm{Ge}$ in the $\mathrm{Si} / \mathrm{SiGe}$ interface and requires uniform growth of the $\mathrm{Si}_{1-x} \mathrm{Ge}_{x}$ layer with precisely controlled composition of $\mathrm{Si}$ and $\mathrm{Ge}$, which is challenging. ${ }^{18-21}$

Here, we present a new strain engineering approach to induce a biaxial tensile strain to the active Si device layer without using an additional epitaxial stressor layer. In this approach, the tensile strain in $\mathrm{Si}$ is naturally created in the process of etching and physical transfer for building flexible $\mathrm{Si}$ devices with high performance on plastic substrates. A strained Si nanomembrane is fabricated by releasing the bilayer of $\mathrm{Si}$ and $\mathrm{SiO}_{2}$ from the host $\mathrm{Si}$ layer of a silicon-on-insulator (SOI) wafer. The oxidation-induced compressive strain in the $\mathrm{SiO}_{2}$ layer of the SOI wafer is activated by forming a suspended structure via the etching of the host $\mathrm{Si}$ that leads to planar expansion of the $\mathrm{Si} / \mathrm{SiO}_{2}$ bilayer and resultantly induces biaxial tensile strain in the top $\mathrm{Si}$ layer. The extent of strain sharing depends on the relative thicknesses of the two layers. The transfer step is inevitably required for the proposed Si devices that well fits this approach to flexible electronics. After transferring the strained Si membrane to the plastic substrate, the fabricated Si devices exhibited a $20-40 \%$ greater charge carrier mobility. The strained $\mathrm{Si}$ membrane can benefit from the well-established SOI electronics and offers an important advantage over the conventional method that uses a $\mathrm{Si}_{1-x} \mathrm{Ge}_{x}$ stressor layer.

\section{EXPERIMENTAL PROCEDURE}

Fabrication of strained Si transistors

The schematic illustrations of whole fabrication steps for strained Si thin-film transistors (TFTs) are displayed in Supplementary Figure S1. SOI wafer was used to fabricate the Si TFTs. The SOI wafer was composed of $0.43-1.5 \mu \mathrm{m}$ top $\mathrm{Si}, 1 \mu \mathrm{m} \mathrm{SiO}{ }_{2}$ and bulk $\mathrm{Si}$. A top $\mathrm{Si}$ layer of SOI wafer was $n^{+}$doped to form ohmic contacts with the source and drain electrodes of the TFTs by implantation with phosphorus (dose $=5 \times 10^{15} \mathrm{~cm}^{-2}$, energy $=15 \mathrm{KeV}$ ). Si layers outside the device parts were etched using $\mathrm{SF}_{6}$ plasma by reactive-ion etching to prohibit the interference of currents flowing into each device. $\mathrm{A} \mathrm{SiO}_{2}$ dielectric layer $(70 \mathrm{~nm})$ was grown by a thermal oxidation process at $900{ }^{\circ} \mathrm{C}$ for $39 \mathrm{~min}$. An opening process was performed to make a via hole between the

\footnotetext{
${ }^{1}$ School of Electrical and Electronic Engineering, Yonsei University, Seoul, Korea and ${ }^{2}$ Department of Nanomechanics, Nano-Convergence Mechanical Systems Research Division, Korea Institute of Machinery and Materials (KIMM), Daejeon, Korea

Correspondence: Dr J-H Kim, Department of Nanomechanics, Nano-Convergence Mechanical Systems Research Division, Korea Institute of Machinery and Materials (KIMM), Daejeon 34103, Korea.

E-mail: jaehkim@kimm.re.kr

or Professor J-H Ahn, School of Electrical and Electronic Engineering, Yonsei University, Seoul 03722, Korea.

E-mail: ahnj@yonsei.ac.kr

Received 5 October 2015; revised 29 December 2015; accepted 18 January 2016
} 


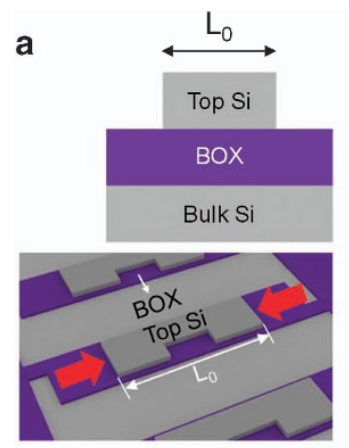

On SOI wafer

b

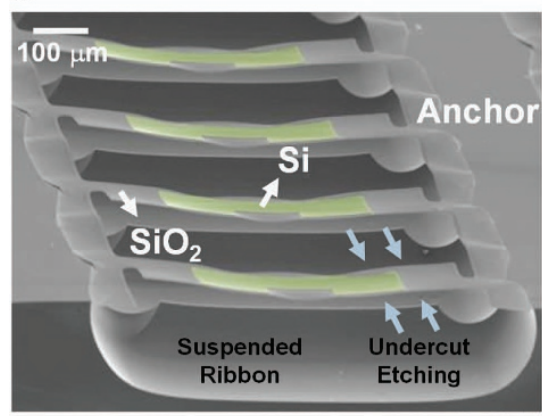

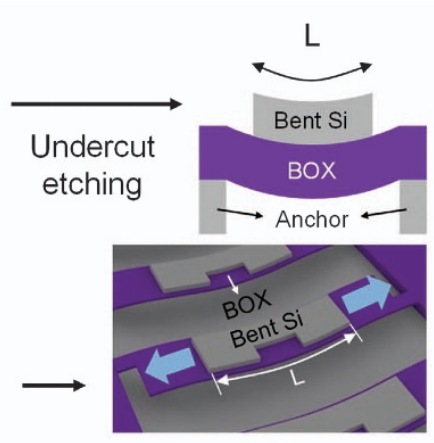

Suspended structure

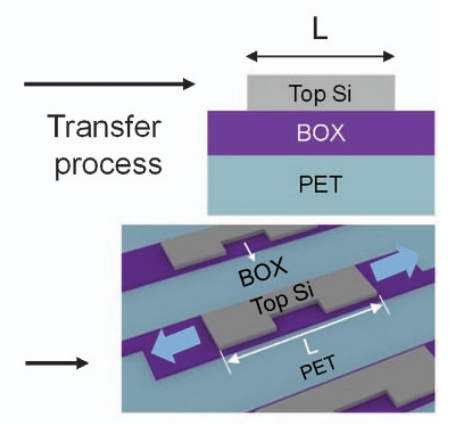

Transferred on PET

d
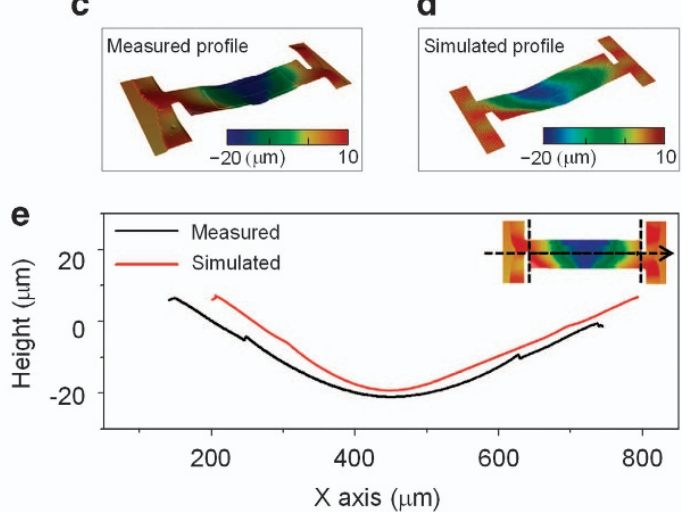

Figure 1 (a) A schematic of the processes used to apply tensile strain to a Si ribbon. (b) A scanning electron microscope (SEM) image of suspended $\mathrm{Si} / \mathrm{SiO}_{2}$ ribbons after removing bulk $\mathrm{Si}$. Visualized height profiles of the suspended $\mathrm{Si} / \mathrm{SiO}_{2}$ ribbon (c) measured by white light interferometer (left) and (d) simulated by finite element method (FEM; right). (e) Height profiles of the center part of suspended $\mathrm{Si} / \mathrm{SiO}_{2}$ ribbons along the longitudinal direction. The black solid curve is for the measured height profile by white light interferometry, and the red solid curve is for the simulated one by finite element analysis (FEA), respectively. BOX, buried oxide; PET, polyethylene terephthalate; SOI, silicon-on-insulator.

sources and drains of the $\mathrm{Si}$ and electrodes by wet etching of $\mathrm{SiO}_{2} \cdot \mathrm{Cr} / \mathrm{Au}$ layers with thicknesses of 5 and $95 \mathrm{~nm}$, respectively, were deposited by thermal evaporation and defined as source/drain and gate electrodes by photolithography.

\section{Roll-based transfer processes}

Patterns of ribbons with a length of $610 \mu \mathrm{m}$ and a width of $160 \mu \mathrm{m}$ were formed using AZ33XT $(10 \mu \mathrm{m})$ followed by a hard baking process at $115^{\circ} \mathrm{C}$ for $3 \mathrm{~min}$. A lateral dry etching process using an inductively coupled plasma reactive-ion etcher $\left(\mathrm{SF}_{6}, 130 \mathrm{sccm}, 50 \mathrm{mTorr}, 35 \mathrm{~min}\right)$ was carried out to remove the bulk $\mathrm{Si}$ underneath the buried oxide (BOX) layer to form suspended device ribbons. A roll transfer process was then used to carry out the reliable transfer of suspended device ribbons with precise control of the transfer load. Roll transfer equipment generally consists of a roller, a load cell, a $z$-axis actuator and a transfer plate as shown in Supplementary Figure S2. The inset shows the schematic illustration of the roll transfer system. The roller is parallel to the transfer plate, and its vertical motion is controlled by two $z$-axis actuators. The exact load applied to the devices is accurately measured by the load cell and is controlled by the input system connected to the actuators. The transfer processes are mainly divided into two steps: picking step and placing step. In the picking step, large-area suspended device arrays held onto the transfer plate by vacuum were placed in contact with the roller that was covered by a polydimethylsiloxane film with an optimized contact load. Suspended device arrays were detached from the wafer and attached to polydimethylsiloxane by van der Waals adhesion force. In this procedure, the angular displacement of the roller was synchronized with the in-plane displacement of the transfer plate to prohibit device arrays from being misaligned by shear stress. In the placing step, the target substrate should be held onto the second transfer plate. Contact between device arrays attached to the roller and a target substrate was made with the optimized load. Adhesives such as NOA 63 (Norland Products Inc., Cranbury, NJ, USA) and SU-8 2005 (Microchem Corp., Newton, MA, USA) were coated on the surface of the target substrate to facilitate a larger adhesion force between the devices and the target substrate than that between the devices and the roller. Through the rolling steps with the synchronized motion of the roller and the plate at $90{ }^{\circ} \mathrm{C}$, picked devices were transferred onto a target substrate. The resultant devices realized by the rollbased transfer technique are shown in Supplementary Figure S3. Large-area device arrays including $\sim 3000$ devices in $4 \times 4 \mathrm{~cm}$ scale were transferred onto plastics with robust operation.

\section{RESULTS AND DISCUSSION}

Figure 1a illustrates the application of self-sustained biaxial tensile strain to $\mathrm{Si} / \mathrm{SiO}_{2}$ ribbon arrays suspended on a wafer by releasing residual compressive stress confined in $\mathrm{SiO}_{2}$. An SOI wafer with a top $\mathrm{Si}$ (100) layer of various thicknesses on a BOX layer was used to fabricate strained Si ribbons. A large volume expansion accompanies the oxidation process of $\mathrm{Si}$ to form the BOX layer because the molar volume of $\mathrm{SiO}_{2}$ is larger than that of $\mathrm{Si}$. However, the volumetric expansion of $\mathrm{SiO}_{2}$ is constrained because it is bonded to the underlying $\mathrm{Si}$ handle wafer, and hence a high residual compressive stress of $0.2-6 \mathrm{GPa}$ is biaxially developed in the BOX layer. ${ }^{22-26}$ Etching out of the underlying handle Si leads to a biaxial expansion of the BOX and, as a result, creates biaxial tensile strain in the top Si ribbon that is tightly bonded to the BOX. The tensile strain in the $\mathrm{Si}$ is determined by equilibrating the force between the $\mathrm{Si}$ and the $\mathrm{SiO}_{2}$. The suspended ribbons are anchored on both edges, and hence the biaxial tensile stress in the ribbons generally generates a boat shape because of 
buckling (Figure 1b). 27,28 The extent and distribution of strain can be evaluated by analyzing the degree of buckling and the deformed shape. $^{29}$

Figure 1c presents the result of a three-dimensional height profile of the suspended $\mathrm{Si} / \mathrm{SiO}_{2}$ ribbon measured by white light interferometry. The deformation of the ribbon was evaluated by measuring the change of transverse and longitudinal length in the $\mathrm{Si}$ and $\mathrm{SiO}_{2}$ regions. Based on the dimensions of the suspended ribbon (Supplementary Figure S4), the residual stress in the BOX layer and the average tensile strain applied to the suspended Si layer were estimated to be $854 \mathrm{MPa}$ and $0.318 \%$, respectively (Supplementary Note S1). To confirm these results, finite element analysis was carried out on a model of the suspended $\mathrm{Si} / \mathrm{SiO}_{2}$ ribbon under the same conditions (Supplementary Note S2); the simulated three-dimensional profile of the suspended ribbon (Figure 1d) was analogous to the measured one. The distance between the center and the edge of the ribbon along the longitudinal direction was $23.89 \mu \mathrm{m}$ in the simulated profile and $26.05 \mu \mathrm{m}$ in the measured ribbon (Figure 1e).

Figure 2a presents finite element analysis results of the distribution of in-plane principal strain on a suspended $\mathrm{Si} / \mathrm{SiO}_{2}$ ribbon. The contour map of strain shows that a tensile strain of $\sim 0.310 \%$ is uniformly applied to the whole area of the table-shaped $\mathrm{Si}$ on $\mathrm{SiO}_{2}$. This measurement agrees with the result of the height profile measurement $(0.318 \%)$. The distribution of strain along the $\mathrm{x}$ and $\mathrm{y}$ axes of a ribbon are displayed in Figures $2 \mathrm{~b}$ and $\mathrm{c}$, respectively, showing different average values of tensile strain $\left(\varepsilon_{\mathrm{x}}\right.$, avg $=0.207 \%$, $\varepsilon_{\mathrm{y} \text {, avg }}=0.271 \%$ ) with a nonuniform distribution. This is because the ribbon is longitudinally and asymmetrically linked to the anchors and constrained by the rigid edges while being transversely free without any constraint (Supplementary Figure S5).

Raman spectroscopy was used to measure the distribution of the induced strain in a suspended $\mathrm{Si}$ ribbon, and the results were compared with the results above. The strain in the Si was directly calculated by observing the shift of a strong Raman peak near $520 \mathrm{~cm}^{-1} .30,31$ Figure 3a presents the contour mapping image of Raman shift for unstrained Si on an unetched region and a strained, suspended Si ribbon that displays a clearly distinguished, uniform strain distribution on two regions. Raman peaks of unstrained Si and strained $\mathrm{Si}$ were observed at 520.46 and $517.98 \mathrm{~cm}^{-1}$, respectively.
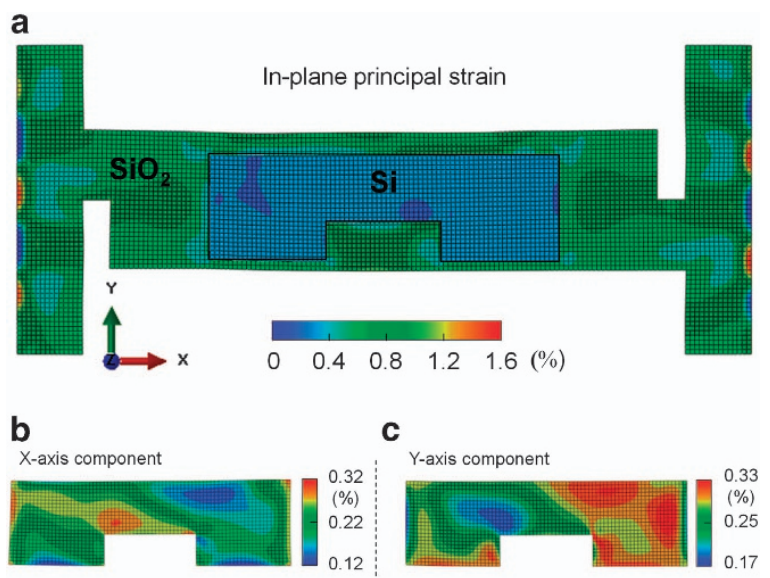

Figure 2 (a) A contour map of the in-plane principal strain distribution on the suspended $\mathrm{Si} / \mathrm{SiO}_{2}$ ribbon simulated by finite element analysis (FEA). The FEA contour maps of (b) normal strain in the $x$ direction (left) and (c) normal strain in the y direction (right) in the suspended Si area.
This image shows good agreement with the simulated one shown in Figure 2a. Figure $3 \mathrm{~b}$ shows Raman spectra that illustrate the change in strain at different conditions: bulk, anchored, suspended and transferred Si. Raman peaks of bulk and anchored Si appeared at $520.46 \mathrm{~cm}^{-1}$, and peaks of suspended and transferred Si ribbons were observed at 517.98 and $518.22 \mathrm{~cm}^{-1}$, respectively. Based on the Raman peak of unstrained $\mathrm{Si}\left(520.46 \mathrm{~cm}^{-1}\right)$, those of the suspended and transferred ribbons were downshifted $(\Delta \omega)$ by 2.48 and $2.24 \mathrm{~cm}^{-1}$ with a tensile strain $\left(\varepsilon_{\mathrm{Si}}\right)$ of 0.316 and $0.286 \%$, respectively, given $b^{31}$

$$
\Delta \omega=b \times \varepsilon_{S i}
$$

In Equation (1), $b$ is the strain shift coefficient of the Si-Si phonon mode on strained $\mathrm{Si},-784 \pm 4 \mathrm{~cm}^{-1}$. Although uniform tensile strain was distributed in the transferred ribbon (Supplementary Figure S6), a slight decrease in the strain value of the $\mathrm{Si}$ ribbon may be created during the transfer to a polyethylene terephthalate (PET) substrate. ${ }^{32}$ When an $\mathrm{Si} / \mathrm{SiO}_{2}$ ribbon is transferred to the PET substrate, it is heated to $\sim 100^{\circ} \mathrm{C}$ to cure the polymer adhesive and improve the bonding strength between the ribbon and the substrate. A cooling process after heating can induce a slight compressive strain on the ribbon because of the higher thermal expansion coefficient $(\alpha)$ of the PET substrate $\left(\alpha_{\mathrm{PET}}=5.9 \times 10^{-5} \mathrm{~K}^{-1}\right)$ than that of $\mathrm{Si} / \mathrm{SiO}_{2}\left(\alpha_{\mathrm{Si}}=2.6 \times 10^{-6} \mathrm{~K}^{-1}, \alpha_{\mathrm{SiO}_{2}}=0.35 \times 10^{-6} \mathrm{~K}^{-1}\right)$. However, we observed that transferred ribbons maintained their original state without detectable deformation in Raman shift for 28 days (Supplementary Figure S7).

The tensile strain applied to the Si layer is controllable by adjusting the stiffness ratio of $\mathrm{Si}$ and $\mathrm{SiO}_{2}$ because the residual strain from the bottom $\mathrm{SiO}_{2}$ layer is more effectively transferred to the thinner $\mathrm{Si}$ layer. To assess the effects of the thickness ratio, $\mathrm{Si} / \mathrm{SiO}_{2}$ ribbons with different Si thicknesses $(0.43-1.5 \mu \mathrm{m})$ and a constant thickness of $\mathrm{SiO}_{2}$ $(\sim 1 \mu \mathrm{m})$ were transferred to a foreign substrate, and the induced tensile strain was measured by Raman spectroscopy (Figure 3c). The specific Raman peak of unstrained, bulk $\mathrm{Si}$ at $520.46 \mathrm{~cm}^{-1}$ showed larger downshifts as $\mathrm{Si}$ thickness decreased from $2.17 \mathrm{~cm}^{-1}$ for $1.5 \mu \mathrm{m}$-thick $\mathrm{Si}\left(\varepsilon_{\mathrm{Si}}=0.277 \%\right)$ to $4.70 \mathrm{~cm}^{-1}$ for $0.43 \mu \mathrm{m}$-thick $\mathrm{Si}$ $\left(\varepsilon_{\mathrm{Si}}=0.568 \%\right.$; Figure $\left.3 \mathrm{~d}\right)$. The measured strain values are in good agreement with those obtained from finite element analysis (Supplementary Note S3). Ultimately, the strain level can be achieved up to $1 \%$ as predicted by the calculation.

A primary purpose for applying tensile strain in $\mathrm{Si}$ is to improve its electronic properties; the strain reduces carrier scattering and the effective transport mass of electrons. ${ }^{33,34}$ The roll-based transfer approach of a strained Si layer can effectively provide important benefits to the development of high-performance strained Si TFTs for large-area flexible electronic applications. ${ }^{15-17,35}$ Figure 4 a shows the key fabrication steps for a strained Si TFT array on a flexible PET film using an automated roll transfer method. All device processes, including doping, oxidation and metallization, were performed on SOI wafers with different top Si thicknesses of $0.43-1.5 \mu \mathrm{m}$ through a similar method to the conventional process. ${ }^{36,37}$ After measuring the pristine characteristics of the device on a wafer, the device array ( $\sim 3000$ transistors on a $4 \times 4 \mathrm{~cm}$ area) was printed on a PET substrate after undergoing the etching and roll transfer processes (Supplementary Figures S2 and S3). As seen in the magnified image, the surface of a strained Si TFT transferred onto a PET substrate looks smooth and clean because the distortion induced by the anchors was relieved by the sliding on the polydimethylsiloxane stamp (Supplementary Figure S8). In addition, no cracks were observed in the transferred devices, particularly on the $\mathrm{SiO}_{2}$ layer. Thus, it is 

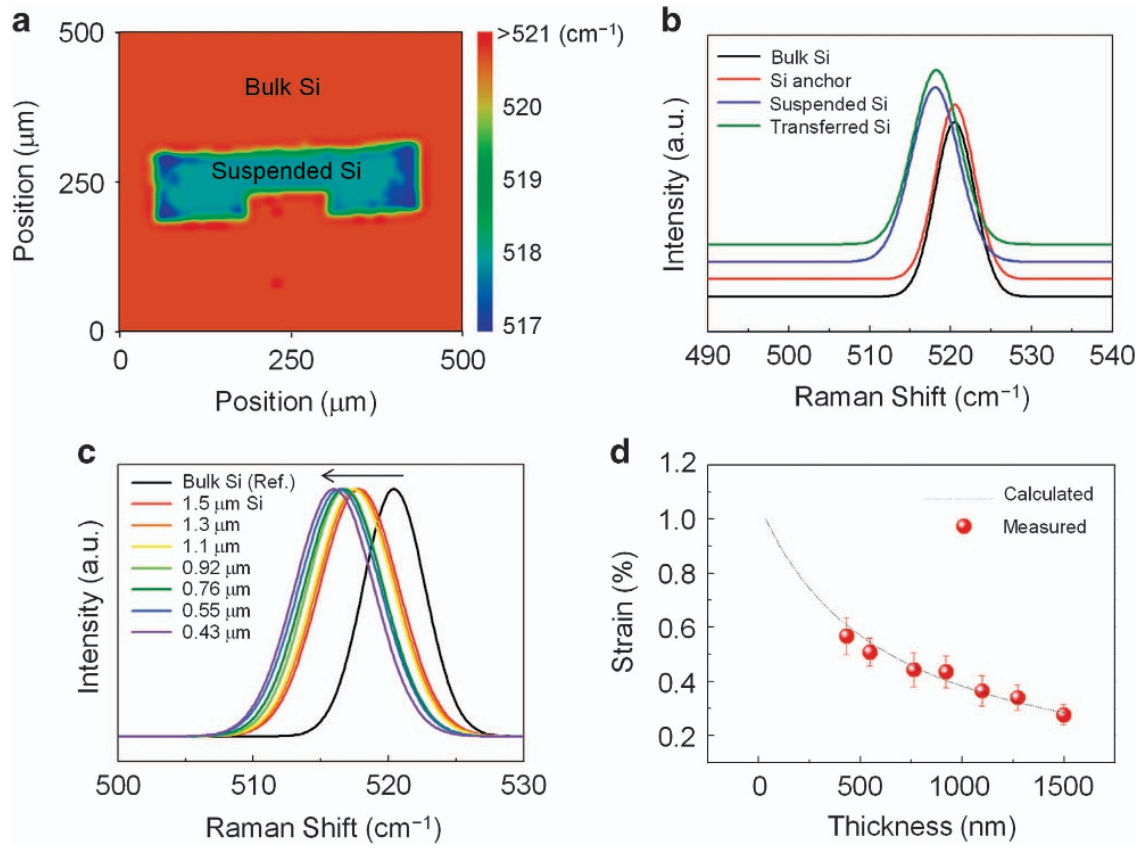

Figure 3 (a) A contour map of Raman peaks in a suspended Si ribbon. (b) Raman spectra of bulk Si (black), Si anchor (red), suspended Si (blue) and transferred Si (green). (c) Raman spectra of suspended Si ribbons with various thicknesses ranging from 0.43 to $1.5 \mu \mathrm{m}$. (d) Calculated and measured strain levels according to the thickness of the top $\mathrm{Si}$ layer in a suspended $\mathrm{Si} / \mathrm{SiO}_{2}$ ribbon.

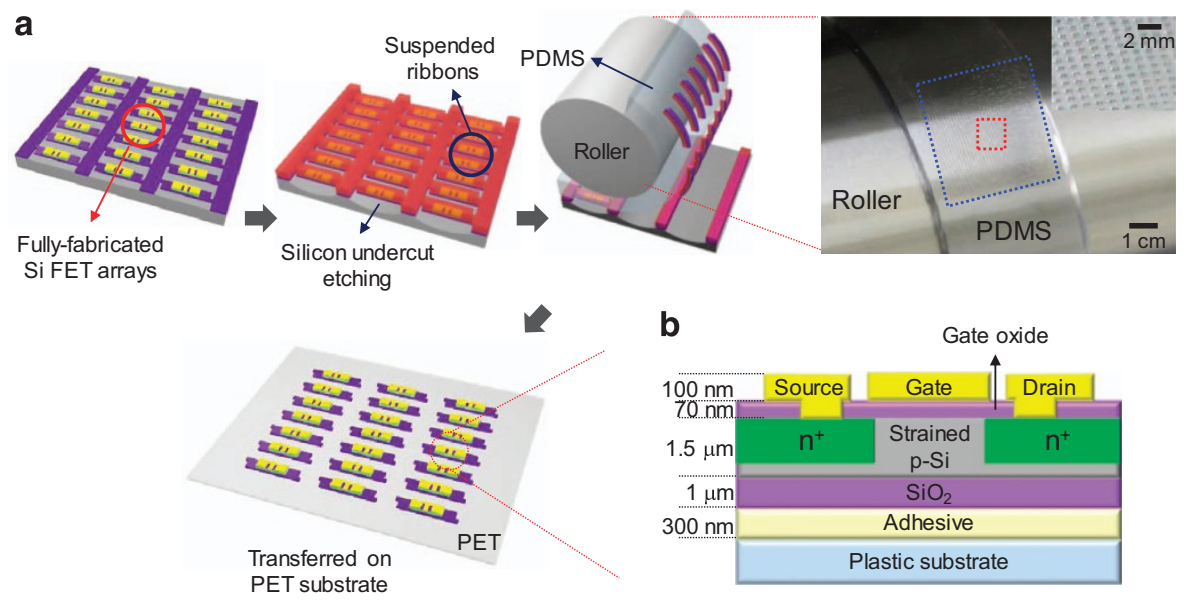

Figure 4 (a) Schematics of the steps to fabricate the strained Si thin-film transistors (TFTs) onto a polyethylene terephthalate (PET) substrate including suspending steps of devices by undercut etching and transfer steps using roll-based transfer printing. (b) An illustration of the cross-sectional view of the strained Si TFT on the PET substrate. PDMS, polydimethylsiloxane.

determined that the thin $\mathrm{SiO}_{2}$ in the device is stable under the strain level of $0.5 \%$ because it forms a good interface with the Si through strong covalent bonds.

In contrast to the $\mathrm{Si} / \mathrm{SiO}_{2}$ ribbon, the induced strain of the $\mathrm{Si}$ channel in the TFT can be affected by additional layers, such as a dielectric layer, and electrodes that are required to produce a top-gated TFT structure (Figure $4 \mathrm{~b})$. The top $\mathrm{SiO}_{2}$ dielectric layer $(\sim 70 \mathrm{~nm})$ grown thermally on $\mathrm{Si}$ can induce strain on the $\mathrm{Si}$, as with the bottom $\mathrm{SiO}_{2}$, and also increase the applied strain on the Si by reducing the overall Si thickness. In contrast, a metal electrode $(\sim 100 \mathrm{~nm})$ deposited on the $\mathrm{SiO}_{2} / \mathrm{Si}$ can attenuate the straining effect of the top $\mathrm{SiO}_{2}$ layer on the $\mathrm{Si}$ layer. It was calculated that the top-gated structures induce a slight increase in tensile strain applied to the $\mathrm{Si}$ layer compared with pristine $\mathrm{Si} / \mathrm{SiO}_{2}$ ribbons (from 0.277 to $0.292 \%$ for $1.5 \mu \mathrm{m}$-thick Si and from 0.568 to $0.584 \%$ for $0.43 \mu \mathrm{m}$-thick Si). These values indicate that the effect of the two additional layers is not great (Supplementary Note S4).

The strained Si devices transferred onto PET displayed significant improvement in device characteristics as shown in Figure 5. The drain current $\left(I_{\mathrm{d}}\right)$, compared with that of the unstrained device on a rigid wafer, increased by $16.8 \%$ for $1.5 \mu \mathrm{m}$-thick Si. $I_{\mathrm{d}}$ then gradually increased with decreasing thickness of the $\mathrm{Si}$, and for $0.43 \mu \mathrm{m}$-thick Si, $I_{\mathrm{d}}$ was $30.5 \%$ greater than that of the unstrained rigid wafer (Figure 5a). The on/off ratio $\left(>10^{5}\right)$ was unaffected by the $\mathrm{Si}$ thickness. $I_{\mathrm{d}}-V_{\mathrm{g}}$ characteristics also exhibited clear increases in drain currents in both linear and saturation regions, indicating the effects of 
a
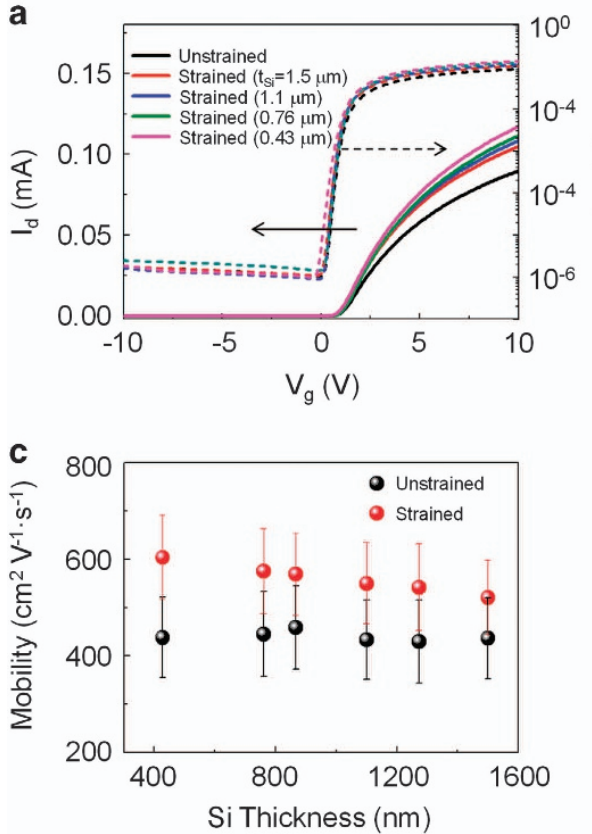

b

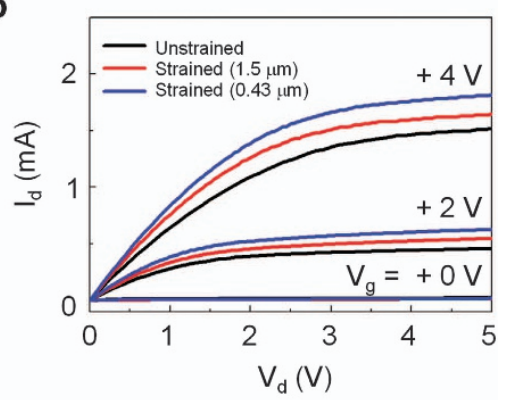

d

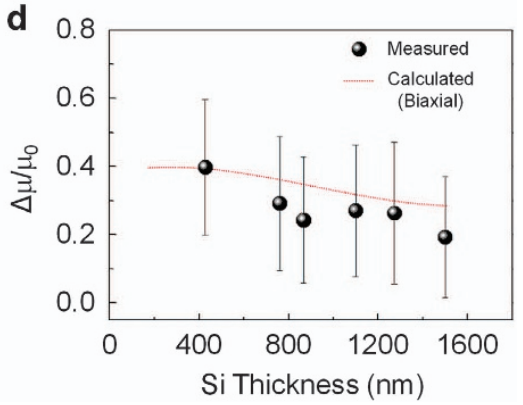

Figure 5 (a) Transfer characteristics of the Si thin-film transistor (TFT) on a wafer and strained Si TFT with various Si thicknesses (0.43-1.5 $\mu \mathrm{m})$ on a polyethylene terephthalate (PET) substrate on a linear scale (line, left y axis) and a log scale (dash, right y axis) for a channel width of $100 \mu \mathrm{m}$ and length of $10 \mu \mathrm{m}$ at a drain voltage of $0.1 \mathrm{~V}$. (b) Output characteristics of the Si TFT on a wafer and strained Si TFT with various Si thicknesses $(0.43-1.5 \mu \mathrm{m})$ on a PET substrate at gate voltages from $0 \mathrm{~V}$ (bottom) to $4 \mathrm{~V}$ (top). (c) Mobility enhancement of strained Si TFTs with various Si thicknesses $(0.43-1.5 \mu \mathrm{m})$ compared with unstrained Si TFTs. (d) Normalized change of mobility of strained Si TFTs with various Si thicknesses $(0.43-1.5 \mu \mathrm{m})$. The red dot curve indicates the calculated results.

a

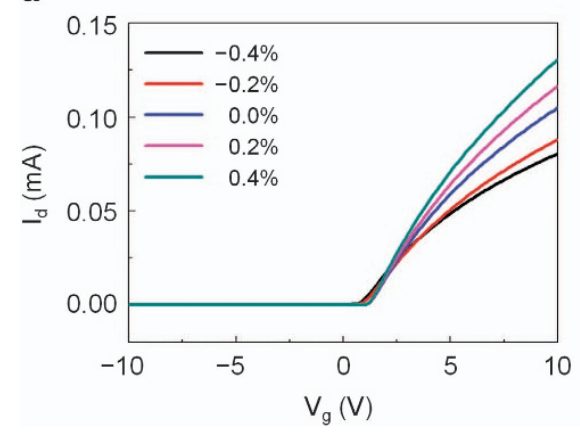

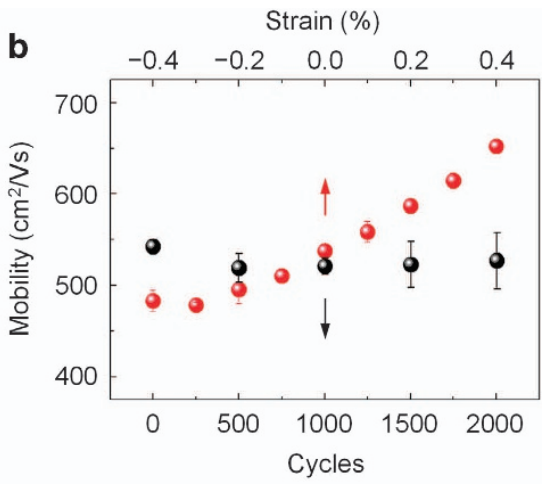

Figure 6 Uniaxial strain effects on electrical properties of strained Si thin-film transistors (TFTs) under bending. (a) Transfer characteristics of strained Si TFTs with a 1.5- $\mu \mathrm{m}$-thick top Si layer under various bending strains. (b) The change in carrier mobility of strained Si TFTs under bending strain and after cycling tests.

strong strain in thin $\mathrm{Si}$ devices (Figure 5b). The average effective mobility, $\mu_{\mathrm{FE}}$, of unstrained $\mathrm{Si}$ TFTs on a wafer before the transfer was calculated as $440.2 \pm 84.9 \mathrm{~cm}^{2} \mathrm{~V}^{-1} \mathrm{~s}^{-1}$ (Supplementary Figures S9 and S10 and Supplementary Note S5). The mobility of the strained $\mathrm{Si}$ devices on PET substrates increased from 520.7 \pm 77.3 to $604.1 \pm 87.4 \mathrm{~cm}^{2} \mathrm{~V}^{-1} \mathrm{~s}^{-1}$ with decreasing thickness of the $\mathrm{Si}$ (Figure $5 c$ and Supplementary Figure S11). The mobility of strained Si TFTs improved by $20-40 \%$ over unstrained Si TFTs owing to the tensile strain of $0.292-0.584 \%$ without a serious increase in the gate leakage current (Supplementary Figure S12). The degree of this enhancement agrees well with the theoretical calculation based on the electronic properties of biaxially strained silicon (Figure $5 \mathrm{~d}$ and
Supplementary Note S6). ${ }^{34}$ The performance of strained Si TFT would be saturated at $40 \%$ with a higher level of tensile strain. ${ }^{40}$

In addition, the enhanced mobility and threshold voltages exhibit a similar tendency as the channel length increased from 10 to $30 \mu \mathrm{m}$ (Supplementary Figures S13-S15). We measured the device performance for 28 days to confirm the stability of strained Si devices after transferring them to a plastic substrate. The strained Si TFTs exhibited stable operation and no strain relaxation under general environmental conditions for the entire duration of the experiment (Supplementary Figure S16) because the residual strain in the $\mathrm{Si}$ devices was induced by the underlying $\mathrm{SiO}_{2}$ layer rather than the PET substrate that has time-dependent relaxation. ${ }^{38}$ 
Figure 6a shows the transfer characteristics of Si TFTs with $1.5 \mu \mathrm{m}$ thickness on PET substrates under various strains. Uniaxial strains between -0.4 and $0.4 \%$ were applied by bending the flexible substrates along the channel direction. Drain currents in the on-state gradually increased with increasing bending strain. This mechanically applied uniaxial strain induced an additional reduction in the effective mass and charge scattering of electrons along the channel direction..$^{39,40}$ As expected, a tensile bending strain of $0.4 \%$ led to a $21 \%$ increase in mobility (from 537.57 to $652.1 \mathrm{~cm}^{2} \mathrm{~V}^{-1} \mathrm{~s}^{-1}$ ). In contrast, a compressive strain of $0.4 \%$ led to a $10 \%$ decrease in mobility because of an opposite influence on the Si channel. This change in mobility against the external strain shows that the TFTs were operating in a stable manner. Even after the repeated bending test to a tensile strain of $0.4 \%$, during which the TFT was bent 2000 times, the strained $\mathrm{Si}$ devices were unscathed without failure (Figure 6b). Thus, these devices demonstrate reliable operation under repeated bending.

\section{CONCLUSION}

Biaxial tensile strain was residually generated in the Si layer of a $\mathrm{Si} / \mathrm{SiO}_{2}$ structure by utilizing the oxidation-induced volumetric strain of the BOX layer in SOI wafers. The induced strain was analyzed by measuring the mechanically stretched configuration of the suspended $\mathrm{Si} / \mathrm{SiO}_{2}$ ribbons by Raman spectroscopy and white light interferometry and confirmed by finite element analysis. The tensile strain in transferred Si TFTs was induced from 0.292 to $0.584 \%$, depending on the thickness ratio of the bilayer of $\mathrm{Si}$ and $\mathrm{SiO}_{2}$. Because the biaxial tensile strain applied to the $\mathrm{Si}$ channel reduced the intervalley scattering and effective mass of electrons, the suspended and transferred Si TFTs showed a $19-40 \%$ increase in carrier mobility as compared with the unstrained devices on a bulk wafer. Furthermore, realization of large-area, strained Si device arrays $(4 \mathrm{~cm} \times 4 \mathrm{~cm})$ were demonstrated by a roll-based transfer onto a plastic substrate.

In conclusion, improved electrical performance was demonstrated in thin Si TFTs on a flexible substrate by strain engineering without relying on the epitaxial stressor, and this process is compatible with the roll-based transfer methods with high productivity. This approach shows promise for strain-engineered large-area flexible electronics with high performance and productivity.

\section{CONFLICT OF INTEREST}

The authors declare no conflict of interest.

\section{ACKNOWLEDGEMENTS}

This work was supported by the National Creative Research Laboratory (2015R1A3A2066337) through the National Research Foundation of Korea (NRF) and the Research Program of Korea Institute of Machinery and Materials (SC 1090).

1 Yu, B., Chang, L., Ahmed, S., Wang, H., Bell, S., Yang, C.-Y., Tabery, C., Ho, C., Xiang, Q., King, T.-J., Bokor, J., Hu, C., Lin, M.-R. \& Kyser, D. FinFET scaling to $10 \mathrm{~nm}$ gate length. Tech. Dig. Int. Electron Devices Meet. 251-254 (2002).

2 leong, M., Doris, B., Kedzierski, J., Rim, K. \& Yang, M. Silicon device scaling to the sub-10-nm regime. Science 306, 2057-2060 (2004).

3 Green, M. L., Gusev, E. P., Degraeve, R. \& Garfunkel, E. L. Ultrathin $\left(\mathrm{SiO}_{2}\right.$ and $\mathrm{Si}-\mathrm{O}-\mathrm{N}$ gate dielectric layers for silicon microelectronics: understanding the processing, structure, and physical and electrical limits. J. Appl. Phys. 90, 2057-2121 (2001).

4 Robertson, J. High dielectric constant gate oxides for metal oxide Si transistors. Rep. Prog. Phys. 69, 327-396 (2006)
5 Frank, D. J., Dennard, R. H., Nowak, E., Solomon, P. M., Taur, Y. \& Wong, H.-S. P. Device scaling limits of Si MOSFETs and their application dependencies. Proc. IEEE 89, 259-288 (2001).

6 Meindl, J. D., Chen, Q. \& Davis, J. A. Limits on silicon nanoelectronics for terascale integration. Science 293, 2044-2049 (2001).

7 Ito, S. Mechanical stress effect of etch-stop nitride and its impact on deep submicron transistor design. Tech. Dig. Int. Electron Devices Meet. 247-250 (2000).

8 Nanver, L. K., Jovanović, V., Biasotto, C., Moers, J., Grützmacher, D., Zhang, J. J., Hrauda, N., Stoffel, M., Pezzoli, F., Schmidt, O. G., Miglio, L., Kosina, H., Marzegalli, A., Vastola, G., Mussler, G., Stangl, J., Bauer, G., van der Cingel, J. \& Bonera, E. Integration of MOSFETs with SiGe dots as stressor material. Solid-State Electron. 60, 75-83 (2011).

9 Ang, K.-W., Lin, J., Tung, C.-H., Balasubramanian, N., Samudra, G. S. \& Yeo, Y.-C. Strained n-MOSFET with embedded source/drain stressors and strain-transfer structure (STS) for enhanced transistor performance. IEEE Trans. Electron Devices. 55, 850-857 (2008).

10 Chui, K.-J., Ang, K.-W., Madan, A., Wang, H., Tung, C.-H., Wong, L.-Y., Wang, Y., Choy, S.-F., Balasubramanian, N., Li, M. F., Samudra, G. \& Yeo, Y.-C. Source/drain germanium condensation for P-channel strained ultra-thin body transistors. Tech. Dig. Int. Electron Devices Meet. 493-496 (2005).

11 Fischetti, M. V. \& Laux, S. E. Band structure, deformation potentials, and carrier mobility in strained Si, Ge, and SiGe alloys. J. Appl. Phys. 80, 2234-2252 (1996).

12 Lee, M. L., Fitzgerald, E. A., Bulsara, M. T., Currie, M. T. \& Lochtefeld, A. Strained Si, $\mathrm{SiGe}$, and Ge channels for high-mobility metal-oxide-semiconductor field-effect transistors. J. Appl. Phys. 97, 011101 (2005).

13 Roberts, M. M., Klein, L. J., Savage, D. E., Slinker, K. A., Friesen, M., Celler, G., Eriksson, M. A. \& Lagally, M. G. Elastically relaxed free-standing strained-silicon nanomembranes. Nature 5, 388-393 (2006).

14 Scott, S. A. \& Lagally, M. G. Elastically strain-sharing nanomembranes: flexible and transferable strained silicon and silicon-germanium alloys. J. Phys. D Appl. Phys. 40, R75-R92 (2007).

15 Yuan, H.-C., Ma, Z., Roberts, M. M., Savage, D. E. \& Lagally, M. G. High-speed strained-single-crystal-silicon thin-film transistors on flexible polymers. J. Appl. Phys. 100, 013708 (2006).

16 Sun, L., Qin, G., Seo, J. H., Celler, G. K., Zhou, W. \& Ma, Z. 12-GHz thin-film transistors on transferrable silicon nanomembranes for high-performance flexible electronics. Small 6, 2553-2557 (2010).

17 Zhou, H., Seo, J. H., Paskiewicz, D. M., Zhu, Y., Celler, G. K., Voyles, P. M., Zhou, W., Lagally, M. G. \& Ma, Z. Fast flexible electronics with strained silicon nanomembranes. Sci. Rep. 3, 129 (2013).

18 LeGoues, F. K. The effect of strain on the formation of dislocations at the SiGe/Si interface. MRS Bull 21, 38-44 (1996).

19 Gallas, B., Hartmann, J. M., Berbezier, I., Abdallah, M., Zhang, J., Harris, J. J. \& Joyce, B. A. Influence of misfit and threading dislocations on the surface morphology of SiGe graded-layers. J. Cryst. Growth 201/202, 547-550 (1999).

20 Koester, S. J., Rim, K., Chu, J. O., Mooney, P. M. \& Ott, J. A. Effect of thermal processing on strain relaxation and interdiffusion in $\mathrm{Si} / \mathrm{SiGe}$ heterostructures studied using Raman spectroscopy. Appl. Phys. Lett. 79, 2148-2150 (2001).

21 Azuma, Y., Usami, N., Ujihara, T., Sazaki, G., Murakami, Y., Miyashita, S., Fujiwara, K. \& Nakajima, K. Growth of SiGe bulk crystal with uniform composition by directly controlling the growth temperature at the crystal-melt interface using in situ monitoring system. J. Cryst. Growth 224, 204-211 (2001).

22 EerNisse, E. P. Stress in thermal $\mathrm{SiO}_{2}$ during growth. Appl. Phys. Lett. 35, 8-10 (1979).

23 Stan, G., Krylyuk, S., Davydov, A. V. \& Cook, R. F. Compressive stress effect on the radial elastic modulus of oxidized Si nanowires. Nano Lett. 10, 2031-2037 (2010).

24 Moselund, K. E., Dobrosz, P., Olsen, S., Pott, V., De Michielis, L., Tsamados, D., Bouvet, D., O'Neill, A. \& Ionescu, A. M. Bended gate-all-around nanowire MOSFET: a device with enhanced carrier mobility due to oxidation-induced tensile stress. Tech. Dig. Int. Electron Devices Meet. 191-194 (2007).

25 Hay, R. S. Developments in Strategic Materials and Computational Design III (John Wiley \& Sons, Inc., Hoboken, NJ, USA, 2012).

26 Seike, A., Tange, T., Sugiura, Y., Tsuchida, I., Ohta, H., Watanabe, T., Kosemura, D., Ogura, A. \& Ohdomari, I. Strain-induced transconductance enhancement by pattern dependent oxidation in silicon nanowire field-effect transistors. Appl. Phys. Lett. 91, 202117 (2007).

27 Resnik, D., Aljančič, U., Vrtačnik, D., Možek, M. \& Amon, S. Mechanical stress in thin film microstrures on silicon substrate. Vacuum 80, 236-240 (2005).

28 Moselund, K. E., Najmzadeh, M., Dobrosz, P., Olsen, S. H., Bouvet, D., De Michielis, L., Pott, V. \& Ionescu, A. M. The high-mobility bended $\mathrm{n}$-channel silicon nanowire transistor. IEEE Trans. Electron Device 57, 866-876 (2010).

29 Gunnars, J. \& Wiklund, U. Determination of growth-induced strain and thermo-elastic properties of coatings by curvature measurements. Mater. Sci. Eng. 336, 7-21 (2002).

30 Wolf, I. D. Micro-Raman spectroscopy to study local mechanical stress in silicocn integrated circuits. Semicond. Sci. Technol. 11, 139-154 (1996).

31 Wong, L. H., Wong, C. C., Liu, J. P., Sohn, D. K., Chan, L., Hsia, L. C., Zang, H., Ni, Z. H. \& Shen, Z. X. Determination of Raman phonon strain shift coefficient of strained silicon and strained SiGe. Jpn. J. Appl. Phys. 44, 7922-7924 (2005).

32 Rochat, G., Leterrier, Y., Fayet, P. \& Månson, J.-A. E. Stress controlled gas-barrier oxide coatings on semi-crystalline polymers. Thin Solid Films 484, 94-99 (2005).

33 Sun, Y., Thompson, S. E. \& Nishida, T. Physics of strain effects in semiconductors and metal-oxide-semiconductor field-effect transistors. J. Appl. Phys. 101, 104503 (2007). 
34 Yu, D., Zhang, Y. \& Liu, F. First-principles study of electronic properties of biaxially strained silicon: effects on charge carrier mobility. Phys. Rev. B 78, 245204 (2008).

35 Sharma, B. K., Jang, B., Lee, J. E., Bae, S.-H., Kim, T. W., Lee, H.-J., Kim, J.-H. \& Ahn, J.-H. Load-controlled roll transfer of oxide transistors for stretchable electronics. Adv. Funct. Mater. 23, 2024-2032 (2013).

36 Kim, T.-i., Jung, Y. H., Chung, H.-J., Yu, K. J., Ahmed, N., Corcoran, C. J., Park, J. S. Jin, S. H. \& Rogers, J. A. Deterministic assembly of releasable single crystal siliconmetal oxide field-effect devices formed from bulk wafers. Appl. Phys. Lett. 102, 182104 (2013)

37 Chung, H.-J., Kim, T.-i, Kim, H.-S., Wells, S. A., Jo, S., Ahmed, N., Jung, Y. H., Won, S. M., Bower, C. A. \& Rogers, J. A. Fabrication of releasable single-crystal siliconmetal oxide field-effect devices and their deterministic assembly on foreign substrates. Adv. Funct. Mater. 21, 3029-3036 (2011).

38 Kubat, J., Rigdahl, M. \& Selden, R. Internal stresses and activation volumes from the stress relaxation behavior of polyethylene at low deformations. J. Appl. Polym. Sci. 20, 2799-2809 (1976).

39 Chen, F., Euaruksakul, C., Liu, Z., Himpsel, F. J., Liu, F. \& Lagally, M. G. Conduction band structure and electron mobility in uniaxially strained $\mathrm{Si}$ via externally applied strain in nanomembranes. J. Phys. D Appl. Phys. 44, 325107 (2011).

$40 \mathrm{Yu}$, D., Zhang, Y. \& Liu, F. First-principles study of electronic properties of biaxially strained silicon: Effects on charge carrier mobility. Phys. Rev. B 78, 245204 (2008)

(c) (i) This work is licensed under a Creative Commons Attribution 4.0 International License. The images or other third party material in this article are included in the article's Creative Commons license, unless indicated otherwise in the credit line; if the material is not included under the Creative Commons license, users will need to obtain permission from the license holder to reproduce the material. To view a copy of this license, visit http:// creativecommons.org/licenses/by/4.0/

Supplementary Information accompanies the paper on the NPG Asia Materials website (http://www.nature.com/am) 